\title{
Saoithín: A Theorem Prover for UTP
}

\author{
Andrew Butterfield ${ }^{1,2}{ }^{*}$ \\ School of Computer Science \& Statistics ${ }^{1}$ \\ Trinity College Dublin \\ Rep. of Ireland \\ Lero: the Irish Software Engineering Research Centre ${ }^{2}$ \\ Andrew.Butterfield@sccs.tcd.ie
}

\begin{abstract}
SAOITHín is a theorem prover developed to support the Unifying Theories of Programming (UTP) framework. Its primary design goal was to support the higher-order logic, alphabets, equational reasoning and "programs as predicates" style that is prevalent in much of the UTP literature, from the seminal work by Hoare \& He [HH98] onwards. This paper describes the key features of the theorem prover, with an emphasis on the underlying foundations, and how these affect the design and implementation choices. These key features include: a formalisation of a UTP Theory; support for common proof strategies; sophisticated goal/law matching ; and user-defined language constructs. A simple theory of designs with some proof extracts is used to illustrate the above features. The theorem prover has been used with undergraduate students and we discuss some of those experiences. The paper then concludes with a discussion of current limitations and planned improvements to the tool.
\end{abstract}

\section{Introduction}

SAOITHÍN ${ }^{1}$ is an experimental proof assistant for the logic used in UTP, supporting a notion of UTP theory, an intuitive prover interface that supports the equational reasoning style that is prevalent in [HH98] and much other UTPrelated literature, with facilities for the user to define the syntax and semantics of their own language constructs. The tool consists of a core equational prover we have developed in concert with a closely-coupled GUI interface. At present its focus is on supporting foundational work in developing UTP, rather than the application of the theory to a "real-world" design problem.

\subsection{Motivation}

We are doing foundational work in the UTP [HH98], which requires formal reasoning with not only predicates, but also predicate transformers: $\mathbf{R 3}(P) \widehat{=}$ $I I \triangleleft$ wait $\triangleright P$ and predicates over predicates: $P=\mathbf{R 3}(P)$. We also need to

\footnotetext{
* This paper emanated from work part funded by Science Foundation Ireland, Projects 07/RFP/CMSF186, 08/RFP/CMS1277, 03/CE2/I303-1

${ }^{1}$ Pronounced "See-heen".
} 
use recursion at the predicate level: $P \widehat{=} \mu Q \bullet F(Q)$, as well as partially-defined expressions: $s \leqslant s \frown\left(t r^{\prime}-t r\right) \equiv t r \leqslant t r^{\prime}$. The logic being used is therefore semi-classical (two-valued logic, but expressions may be undefined) and of least 2nd-order. In addition, tool support for foundational work in UTP requires the ability to easily describe new language constructs, which can themselves be treated just like predicates, in keeping with the "programs are predicates" philosophy [Hoa85] of UTP.

\section{$1.2 \quad$ Structure}

We next discuss related work $(\S 2)$ in order to better justify our decision to "grow our own" theorem proving assistant. We then proceed to look at the logic $(\S 3$, type-system ( $(4)$, user defined language support ( $(5)$, proof procedures $(\S 6)$, and law matching $(\S 7$, with emphasis on the underlying foundations. We then discuss useability and experience ( $(8)$ and finally finish with future work and conclusions $(\S 9)$.

\section{Related Work}

There are a lot of theorem provers in existence, of which the most prominent feature in [Wie06]. Of these, the most obvious candidates for consideration for UTP prover support are Isabelle/HOL[NPW02], PVS[Sha96], and Coq [BC04]. They are powerful, well-supported, with decades of development experience and large active user communities. They all support higher-order logic of some form, with a command-line interface, typically based around tactics of some form. All three require functions to be total, but support some kind of mechanism for handling partial functions (e.g. dependent types in PVS). Their reasoning frameworks are based on some form of sequent calculus, and do not support equational reasoning in a native fashion.

There has been work done on improving the user interfaces of the above theorem provers. An interesting example was "proof by pointing" [BKT94] for CoQ which allowed the user to select a subterm, whereupon it would generate and apply a tactic based on the subterm's top-level operator. Whilst proof-by-pointing is not supported in more recent versions of CoQ, it has been incorporated into "Proof General" [Asp00], a general purpose user interface for theorem provers, built on top of Emacs. It supports Isabelle and Coq, among others, and is basically a proof-script management system. In essence it supports the commandline tactics of the provers, allowing the user to edit proof scripts at will, whilst maintaining prover consistency behind the scenes.

Within the UTP community, there has been considerable work using ProofPower-Z to build models of UTP theories in Z in order to mechanise proofs. Early work looked at deep embedding into $\mathrm{Z}$ of an imperative language whose semantics were given using UTP [NW06]. Work extending this to a mechanisation of UTP itself was also undertaken, driven by a desire to mechanically verify the semantics of Circus [OCW09]. Recent work has looked at re-working the mechanisation of 
UTP in order to better support the hierarchical nature of UTP theory building [ZC09], where alphabetised predicates are restricted to relations, then designs, and so on. Some support for Z-like theories from UTP (such as Circus) can be found as extensions implemented in the Community Z Tools project [MU05].

Whilst all of good pedigree, CoQ, Isabelle/HOL, ProofPower-Z and PVS all have in common that they work best when used in the manner for which they were designed - in none of these cases does this manner match the way we wish to work in UTP, as described in the introduction. Ironically, the key inspiration for the design of SAOITHín came not from the above provers, but instead from the one provided as part of the RAISE Development Method [GHH $\left.{ }^{+} 95\right]$. That theorem prover had mechanisms for selecting sub-expressions and identifying applicable laws for that sub-expression, a feature very close to that required for the proof style that SAOITHÍN supports.

\section{The Logic}

A subset of the syntax of the predicate logic of SAOITHÍn is shown below:

$$
\begin{gathered}
P, Q \in \text { Pred }::=\text { True } \mid \text { False }|\neg P| P \wedge P|P \vee P| P \Rightarrow P \mid P \equiv P \\
|A| \forall \vec{v} \bullet P|\exists \vec{v} \bullet P| M \mid P[\vec{e} / \vec{v}] \\
e, f \in \text { Expr }::=v|M| \text { expressions using }+, \times, \leqslant, \cup, \frown \ldots, v \in \text { Var }
\end{gathered}
$$

In addition to the usual propositions and quantifiers, we also have atomic predicates ( $A$, boolean-valued expressions), meta-variables denoting arbitrary predicates or expressions $(M)$, and an explicit substitution notation $P[\vec{e} / \vec{v}]$. The predicate meta-variables allow us to write conjectures and laws true for arbitrary predicates, while explicit substitution is required as many definitions in UTP use it in such an explicit manner. The axiomatisation being used is one for equational logic [Tou01], extended to support 2nd-order features, with the inference rules (Liebniz, etc.) effectively being implemented by the law matcher $(\S 7)$.

Predicates in SAOITHÍn can participate in a number of roles, of which the most basic are laws and conjectures. Law are either asserted to be true (axioms), or are conjectures that have been proven, and are now theorems. A theorem is then a conjecture coupled with a proof. Some of the axioms/inference rules of predicate calculus have side-conditions, but given explicit meta-variables and substitutions, we find we can no longer treat side-conditions as statically checkable at law-application time, necessitating an explicit representation:

$$
\begin{aligned}
& V \in \text { VSet }::=\mathcal{P} \text { Var } \\
& s c \in \text { Side }::=\text { True }|M=V| M \subseteq V|M \pitchfork V| \text { c. } M \mid \bigwedge\left\{s c_{1}, \ldots, s c_{n}\right\}
\end{aligned}
$$

We read c. $M$ as asserting that $M$ is a condition (no dashed free variables), and $M \rtimes V$ means the free variables of $M$ are disjoint from $V$. The use of side-condition expressions is discussed further in $\S 7.3$. 
The basic unit of work in SAOITHÍN is in fact a predicate coupled with a side-condition and from here on we use the terms conjecture and law to refer to such pairs. So we can view a SAOITHín theory, as a named collection of named conjectures, theorems and laws: ${ }^{2}$

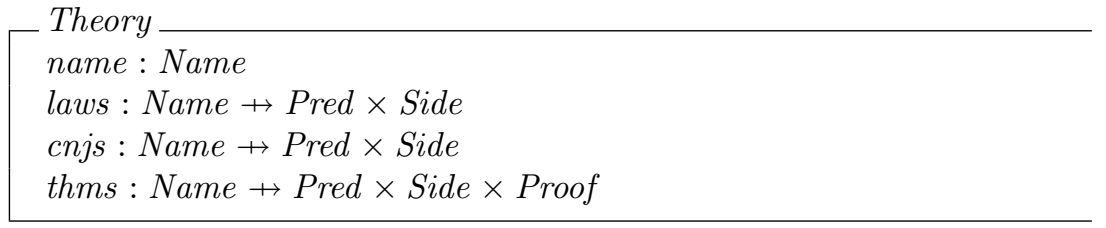

Once a conjecture has been proven it is moved from conjectures to theorems with the same name.

At present, SAOITHÍN is very much an experimental tool, intended in the first instance to support foundational work in the UTP. As a consequence of this, the axioms and inference rules have not, in the main, been hard-coded, but instead the user is free to add their own axioms. This clearly is very dangerous, but does support experimentation. For example, two axiomatisations of predicate calculus have been developed based on [MS89] and [GS94], and initial experimentation suggests that the axiomatisation in the latter leads to easier proofs, due to less choice being available at each step. Versions of SAOITHín that disallow user addition of axioms have been implemented for use in teaching, and we envisage future versions of the tool being able to work in different "user-experience" modes.

The interface to the logic presented to the user is, in the main, based on an ASCII-based concrete syntax, that imitates the mathematical syntax as far as is practicable. This ASCII syntax is used for both input and output. For example, the predicates

$$
[(\exists c \bullet D \wedge L) \Rightarrow S] \quad P[\text { TRUE } / o k]
$$

are written ASCII-style as:

$$
[[(\text { exists } \mathrm{C} @ \mathrm{D} \wedge \mathrm{L}) \Rightarrow \mathrm{S}]] \quad \mathrm{P}[\mathrm{TRUE} / / \text { ok }]
$$

Similarly, side condition $\bigwedge\{Q \subseteq\{a, b\}, e \not\{b, c\}\}$ is written as

$$
\text { coverP } Q \text { a,b ; notinE e b,c }
$$

An almost complete theory (less theorems) can be input and output as a ASCII text file, whose extension is .uttxt (UTP Text). The file is structured as a series of sections, each flagged by a header keyword. The first collection of sections, between SYNTAXFROM and ENDSYNTAX keywords provide information to support the parser. The remaining sections provide semantics information. The first line of such text files is the theory name, optionally followed by a version number. So a simple theory defining some laws and conjectures about conjunction and disjunction could be written as:

\footnotetext{
${ }^{2}$ We use $\mathrm{Z}$ schema notation here for convenience - we are not presenting a formal $\mathrm{Z}$ model of UTP theories.
} 


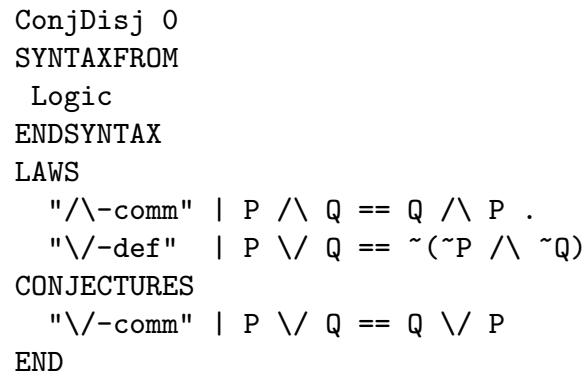

\section{Types}

We have a fairly simple notion of types with booleans and integers making up basic types, a basic type Env that denotes a program environment $($ Name $\rightarrow$ Value), type variables, and then the capacity to build up set/sequence/map/free types on top of these:

$$
\begin{aligned}
& T \in \text { Type }::=\mathbb{B}|\mathbb{Z}| \text { Env } \quad \text { Base Types } \\
& |\tau| ? \quad \text { Type variables } \\
& |\mathcal{P} T| T^{*}\left|T^{+}\right| T \times \cdots \times T \quad \text { Composite types } \\
& \text { | } T \rightarrow T|T \rightarrow T| T \leftrightarrow T \quad \text { Function types } \\
& \left.\left|n m \bullet V^{\prime}\right|^{\prime} \ldots{ }^{\prime}\right|^{\prime} V \quad \text { Free Types } \\
& V \in \text { Variant }::=n m\langle\langle T \times \cdots \times T\rangle\rangle \quad \text { Free Type Variant } \\
& n m \in \text { Name Names }
\end{aligned}
$$

The type-system supports Hindley-Milner style polymorphism, and, for simplicity, treats powerset, sequence and function types as distinct ${ }^{3}$. At present the main role played by types in the prover is to limit the search for applicable laws to those that match the types of expression involved. To this end a type-inferencing algorithm is used to associate types with all expressions. It uses user-supplied information about the types of named functions to deduce the relevant types for entire expressions. This information is stored in a table matching function names to their types, which of course has to be an additional component of a theory:

$$
\begin{aligned}
& \text { Theory } \\
& \ldots \\
& \text { types }: \text { Name } \rightarrow \text { Type }
\end{aligned}
$$

Types have an ASCII syntax as well, as exemplified by this example:

$$
\mathbb{Z} \times \mathcal{P}\left(\tau^{*}\right) \rightarrow \mathbb{B} \quad \mathrm{Z} \times \mathrm{P} \text { t* } \rightarrow \mathrm{B}
$$

We can introduce types into a UTP textfile using a TYPES section, so for example, from a theory of arithmetic, we might have:

\footnotetext{
${ }^{3}$ We could add axioms to a theory relating type-assertion predicates if we wished to link these types.
} 


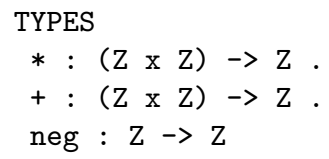

\section{Adding Language}

The main purpose of UTP is to allow the construction, comparison and connection of theories about a variety of modelling, specification and programming languages. This requires us to be able to specify two key aspects:

1. The types of observations of such systems that we wish to discuss, captured by the notion of observation variables;

2. A description of the language under study: both its syntax and semantics.

\subsection{Alphabets}

A key feature of UTP is its use of alphabetised predicates, where the alphabet is a collection of typed observation variables. At present, the support in SAOITHÍN is limited to theories with fixed alphabets, such as the standard reactive systems theories, or theories where all program variables are encapsulated in a single state : Name $\rightarrow$ Value observation. A proposal to broaden this to cover varying alphabets is under consideration for a future revision of the prover. At present, we simply record the (fixed) set of observation variables and their types in a table:

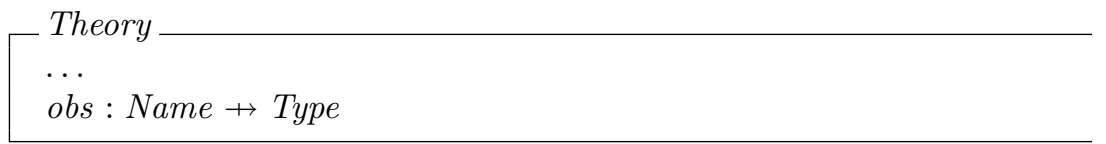

The variables listed in the domain of objs are "known" to the theorem prover and this influences the operation of the matcher. This information is also fed into the type-inferencing system, complementing the types information. We can specify this in the text file, as in the following example for a theory of designs:

OBSVAR

ok : B .

ok' : B

\subsection{User-defined Language Constructs}

In order to be able to describe a theory about the language, we need to be able to describe the language, so we provide facilities to allow the specification (to SAOITHÍn) of the syntax of language constructs.

First, we note that the expression language is easily extendible as the parser automatically converts token streams of the form $n m e$ into the application of 
(function) $n m$ to expression argument $e$. However their effective use often requires the user to give them a type, entered in the types table. New infix operators however are not automatically parsed, and need to be declared in advance, using a simple form associating an operator name with its precedence and associativity. This infix information is stored in a table in the theory:

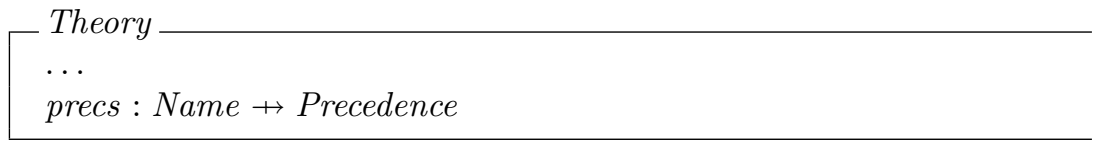

A predecence is a pair of a number and an associativity (None/Left/Right), with higher numbers denoting tighter binding strengths ${ }^{4}$. In a theory of designs, we want to introduce $\vdash$ as an infix operator so we can declare it in a PRECEDENCES section:

PRECEDENCES

I- 55 None

A user-defined language construct is an interleaving of existing forms (variables, expressions, types, predicates) with new tokens, including also some list forms with specified delimiters and separators. So a theory needs to contain a table listing syntax specifications:

$$
\left[\begin{array}{l}
\text { Theory } \\
\ldots \\
\text { lang }: \text { Name } \rightarrow \text { SynSpec }
\end{array}\right.
$$

There is a simple ASCII syntax for defining new language constructs, basically an interleaving of keywords V (variable), T (type), E (expression) and P (predicate), keysymbols * (list) and \# (counted-list) with arbitrary syntactical elements. The keysymbols follow a keyword and are themselves followed by a token denoting a separator. So E*, denotes a comma-separated list of expressions, wherease

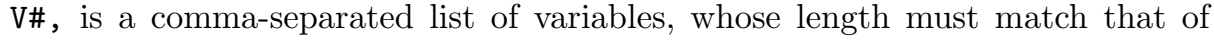
any other list present also defined using \#. A theory of designs and imperative programming might declare its syntax as follows:

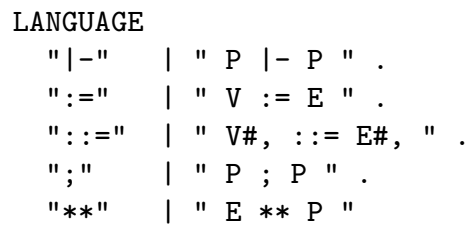

(Here $::=$ is intended to be simultaneous assignment). All language constructs so specified are considered as new instances of predicates, and so can themselves appear and be parsed as language elements, so allowing easy nesting of

$\overline{{ }^{4} \text { Currently } \wedge}: 80, \vee: 60, \Rightarrow: 30, \equiv: 20$ 
such constructs. Any specifications that define an infix operator can also have a precedence declaration.

There are no extra facilities provided to describe the semantics of userconstructs, as such laws are simply provided by the user as appropriate axioms in the theory. For our design theory we might give the semantics of $\vdash$ as follows:

LAWS

"DEF |-" | P |-Q $==$ ok $\bigwedge P \Rightarrow$ ok' 八 $Q$

Note these axioms can be written using the full predicate calculus language shown here, and hence cannot be considered as some form of conservative extension of pre-existing axioms. In addition, for any law regarding a user-construct to be useable, there must be a non-empty collection of laws satisfying the following conditions:

1. Each law has a name prefixed with "DEF $\sqcup$ uname", where uname is the name in lang of the user-construct.

2. Each law has the form $L H S \equiv R H S$ where $L H S$ is an instance of the uname construct.

3. Any instance of the language construct must match at least one of these laws.

4. The RHS s must not mention the construct explicitly, nor should it be possible to construct a cycle via mutual recursion with other user-constructs.

The reason for this set of restrictions is because these laws will be used to automatically expand language definitions "under the hood" in order to evaluate free variables and side-conditions $(\$ 7.2)$. They do not preclude our introducing laws with language recursion, provided their names do not start with "DEF $\sqcup$ uname".

Having introduced our language constructs we then will want to give their semantic definitions as axioms, posit some conjectures, and hopefully prove them to be laws of the language. However, the parser needs to know about language syntax before the rest of the theory text file can be parsed, as we are introducing new lexical elements. So every UTP text file has to have a syntax preamble, immediately after the first line gving the theory name. The preamble has the following format:

SYNTAXFROM

list of zero or more theory names, separated by spaces optional LANGUAGE and PRECEDENCES sections in any order ENDSYNTAX

The theories listed are those with syntax sections defining language constructs used in this theory in addition to the ones it introduces itself.

\section{Proofs}

Given a conjecture in a theory, any attempt to prove it will take place in a general proof context, adopting one of a number of available strategies, and making use of a variety of builtin proof procedures. In general, we do not work with a single 
theory, but rather a stack of them: the bottommost are the most fundamental and general whilst theories higher up the stack tend to be more specific and higher-level. The key idea is that a proof of a conjecture can depend on its own theory, as well as material from theories below it in the stack. The stack concept is provided to assist in the encapsulation of theories, and for this reason circular dependencies are not allowed.

When initially started, SAOITHÍN has a proof stack with a single entry, the _ROOT theory, defining the precedences of the propositional infix connectives. The user can then load up the desired theories, and the current state of the stack is then displayed in the application's main ("top") window:

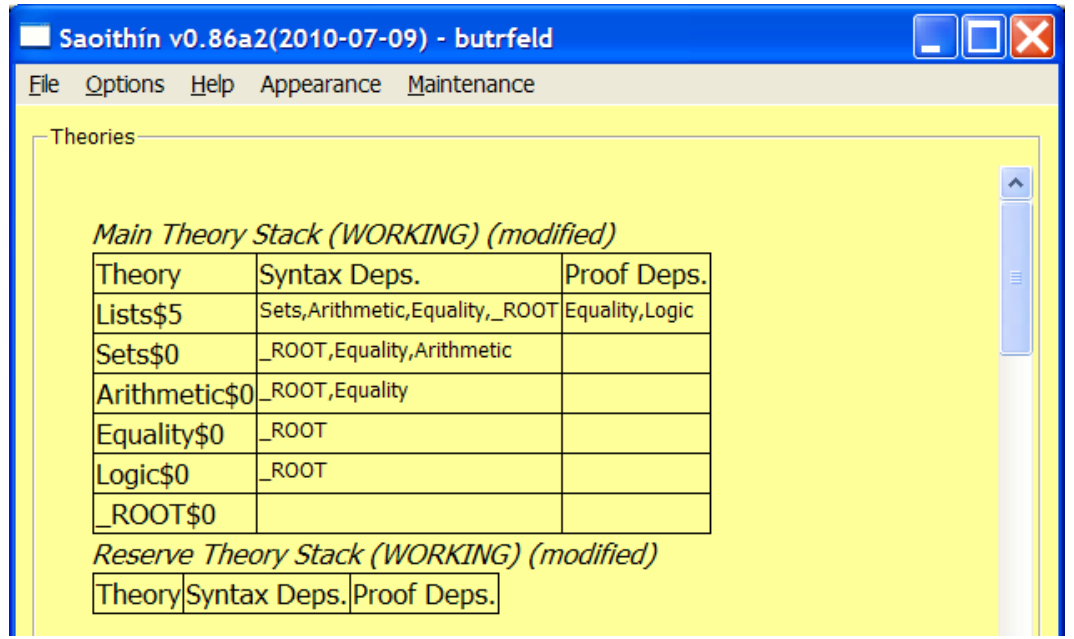

This stack state is saved into a file upon exiting the application, and restored automatically upon re-entry. Double-clicking on a theory name opens up a tabbed window to allow theory components to be inspected:

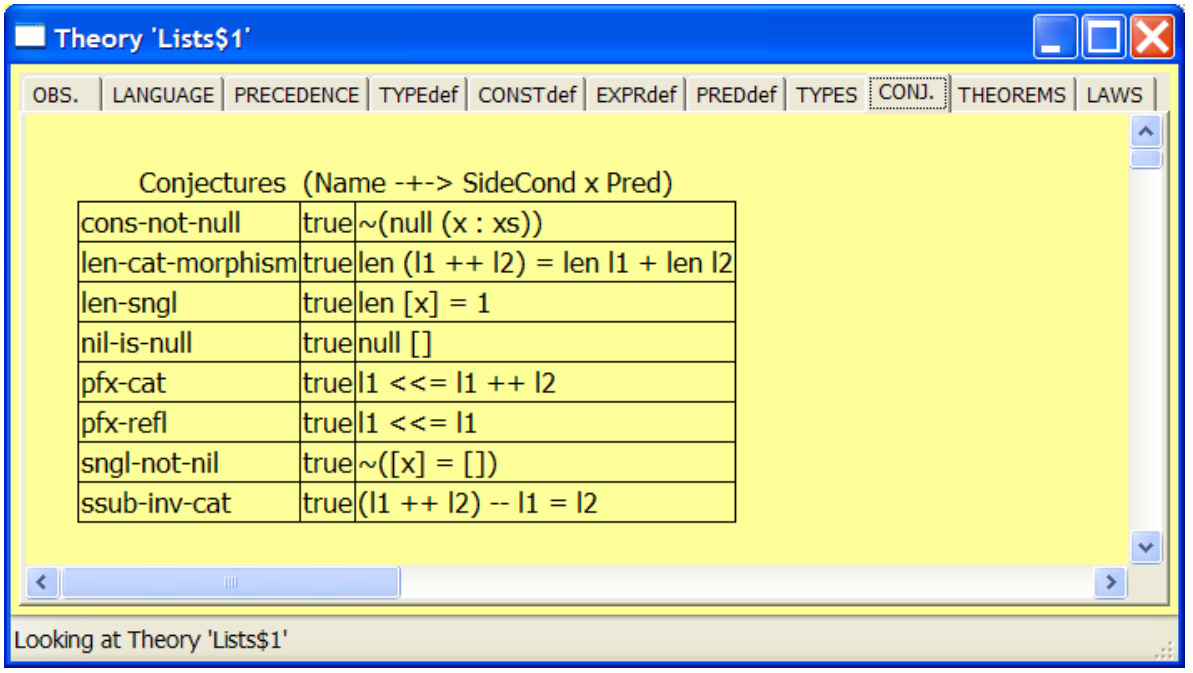


There are a number of strategies we can employ to prove a goal conjecture $G$, based on its top-level structure:

Reduce/Deduce We can simply attempt to take $G$ and use proof procedures to transform it to True (or equivalently, an existing law).

LHS-to-RHS If goal $G$ has the form $L \equiv R$, we can try to transform the LHS $L$ until it is equivalent to $R$ (we could also try RHS-to-LHS).

Reduce-Both Again, for the form $L \equiv R$, a way to proceed might be to try and transform both sides into something equivalent.

Law-Reduce Rather than starting with the conjecture, we start with an instantiation of an existing law, and transform that until equivalent to our conjecture. This strategy is the basis for inductive proofs in SAOITHÍn.

Assume-then-... If the conjecture has the form $A \Rightarrow C$, we can assume the LHS antecedent $A$ as a law, adding it to the proof context, and have it available to assist in proving the consequent $C$ using some other appropriate strategy.

SAOITHín supports the above strategies, and will support more in the future. In general, the chosen strategy may modify both the conjecture and its proof context in some way, and will have a completion criteria that depends on that modification. We shall now discuss some of the concepts just introduced in a little more detail.

Double clicking on a conjecture starts a proof by opening up a proof window. From the Setup menu a strategy can be chosen, for example Reduce:

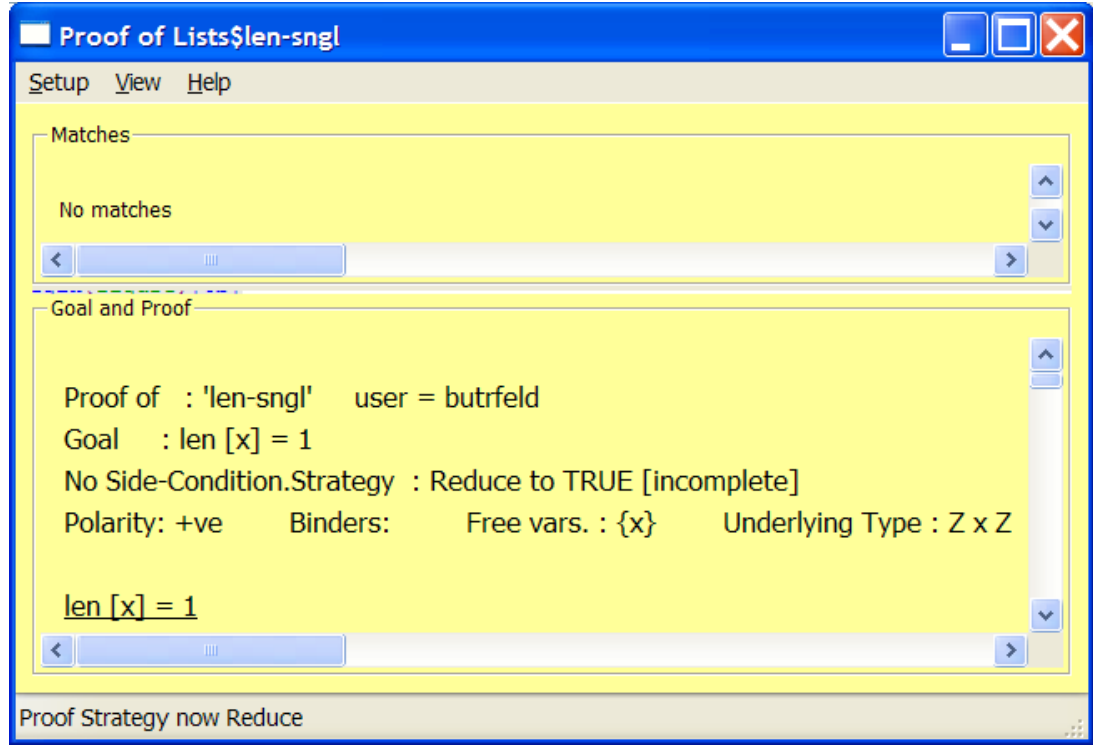

In this case the entire goal appears, with the focus, currently the whole goal, underlined. Given that all atomic predicates are expressions of boolean type, we find more useful typing information to be the notion of underlying type: the types 
of the sub-expressions just below the top-level of the atomic predicate. As the goal is an atomic predicate comparing two integers, we see that its underlying type is $\mathbb{Z} \times \mathbb{Z}$. If attention moves down into sub-expressions, then the full type of that component is displayed, as the notion of underlying type only applies to atomic predicates at the top-level.

\subsection{Transformations}

We mentioned taking a goal and transforming it in some way. As SAOITHÍN favours the use of equational reasoning, such transformations generally involve replacing the goal, or a sub-part of it, by a logically equivalent expression. So a proof becomes a chain of equivalences linking the initial goal to one that is equivalent to the required final predicate, the specifics of which are of course strategy-dependent.

The primary mechanism for effecting such transformations is a process of selecting a sub-part of the current goal (the "focus") and then applying the desired rule. The rules available for application are dependent on the focus, but always include the option to match it against the laws in all the currently accessible theories. The details of this matching is discussed later $(\S 7)$, and for now we give a brief survey of other builtin transformers that can be used:

Tidying Basically a collection of builtin operations to flatten and sort predicates whose top-levels are disjunctions and conjunctions.

Simplify Effectively constant-folding, doing fairly obvious propositional simplifications.

Normal Forms Converting predicates to either disjunctive or conjunctive normal form

Application Reducing applications of abstractions to arguments ( $\beta$-reduction) or applying explicit substitutions to the underlying predicate.

Quantifiers Specifying explicit $\alpha$-substitution, or existential witnesses. If using the Law-Reduce strategy, then we can also strip out top-level universal quantifiers, provided the focus is the whole goal.

Splitting Given long conjunction/disjunctions, it is often useful to be able to restructure them into specific groups in order to allow certain laws to become applicable.

Most of these can be invoked by a single key shortcut (see Help menu in Proof Window)

A proof is complete when goal transformation makes it "equivalent" to an end-point predicate as determined by the strategy in use. The notion of equivalence we use is that of a slight generalisation of $\alpha$-equivalence, that flattens nested quantifiers of the same type, so that the following are considered equivalent:

$$
(\forall x \bullet \forall y \bullet P) \quad(\forall x, y \bullet P) \quad(\forall y, x \bullet P) \quad(\forall y \bullet \forall x \bullet P)
$$




\subsection{Shorthands}

A useful facility in SAOITHín is the ability to define meta-variables as shorthands for longer, more complex predicates. Examples of this in UTP include the definition of $J$ in the reactive theory [HH98, Chp.8, p208]:

$$
J \widehat{=}\left(o k \Rightarrow o k^{\prime}\right) \wedge w_{a i t}^{\prime}=\text { wait } \wedge t r^{\prime}=t r \wedge r e f^{\prime}=r e f
$$

These definitions can be made as part of a theory, or can be introduced on the fly during a proof to replace a long predicate by a shorthand. During a proof it is then possible to replace such a meta-variable by its definition, or even to recursively expand all such shorthands in a predicate - even if the shorthands are themselves mutually recursive. To support this facility, we must add tables to a theory to record these shorthands, which can be introduced for type and predicate meta-variables, as well as regular variables:

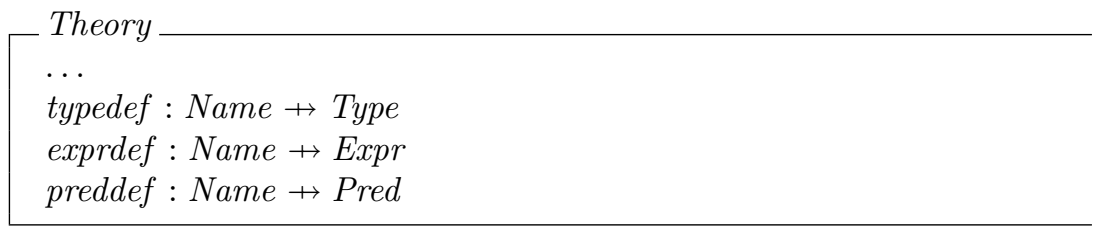

All of the names in these tables are "known", which affects how they participate in law matching. These tables also have another use to ensure the soundness of certain strategies, as detailed below.

\subsection{Assumptions}

The "Assume-" strategies are an implementation of the Deduction Theorem:

$$
\Phi \vdash A \Rightarrow C \quad \text { iff } \quad \Phi, A \vdash C
$$

where the antecedent is elevated (temporarily) to a law for the purposes of this proof alone. However we need to be careful here [GS94, p72], to ensure that all meta-variables in the "assumption-now-law" can only match against themselves - in essence any such variables in $A$ above must be temporarily restricted to only stand for themselves. We create a temporary theory on the top of the theory-stack, and add in as new laws, the antecedent $A$, suitably decomposed (e.g.):

$$
\begin{gathered}
\Phi \vdash\left(A_{1} \wedge A_{2} \wedge A_{3}\right) \Rightarrow\left(\left(A_{4} \wedge A_{5} \wedge A_{6}\right) \Rightarrow C\right) \\
\downarrow \\
\Phi, A_{1}, A_{2}, A_{3}, A_{4}, A_{5}, A_{6} \vdash C
\end{gathered}
$$

We then use the meta-variable tables just introduced in $\S 6.2$, adding in entries of the form $M \widehat{=} M$ for every "unknown" meta-variable in $A$, and finally renaming 
all the $M$ in these tables, $A_{i}$ and $C$ to be fresh, so as to avoid clashes with other laws. This effectively prevents the law matching from binding these metavariables to anything else during the proof. Once the proof is complete, the temporary theory is removed.

\subsection{Induction}

The current support for induction is via the Law-Reduce strategy, using the appropriate induction axiom, instantiated appropriately with the conjecture. This is then simplified until the the goal is itself reached. For example, we may have the following inductive axiom for natural numbers:

$$
M[0 / x] \wedge(\forall y \bullet M[y / x] \Rightarrow M[y+1 / x]) \Rightarrow \forall x \bullet M
$$

(Note that the assumption here is that $x$, if free in $M$ is a natural number). Given addition defined recursively on its left argument, we may wish to prove that $n+0=n$, for all natural $n$. If we invoke the Induction strategy in SAOITHÍN, and upon selecting the above induction axiom, and identifying $n$ as our induction variable, a goal is generated by substituting our conjecture for $M$, and $n$ for $x$, to obtain:

$(n+0=n)[0 / n] \wedge(\forall y \bullet(n+0=n)[y / n] \Rightarrow(n+0=n)[y+1 / n]) \Rightarrow \forall n \bullet(n+0=n)$

Switching to an Assume strategy here doesn't help as we want to manipulate the antecedents. Successive transformations, applying substitutions and using the definition of + , plus other axioms associated with the natural numbers, allow us to reduce the antecedents to true, leaving

$$
\forall n \bullet(n+0=n)
$$

Because we are reducing from an instance of an axiom, this is a theorem, so we can strip off the top-level universal quantification to get our original conjecture:

$$
n+0=n
$$

At present SAOITHÍN requires us to handle induction proofs as one lump as just illustrated, but future strategy/case-handling enhancements will make it possible to do a more "traditional" proof, where the base and inductive cases are effectively proven separately.

\subsection{Caveat Emptor}

Before moving on to look at the law matching facilities we need to point out an important aspect of the transformations discussed in this section: they have built-in to them certain assumptions regarding such notions as $\alpha$-equivalence, the associativity, commutativity and idempotence of conjunction and disjunction, and implication trading, to name but a few. It is therefore important that any axioms introduced in theories do not conflict with these internal assumptions regarding the $\operatorname{logic}{ }^{5}$.

\footnotetext{
${ }^{5}$ This is in fact an argument for having a built-in logic theory that is compatible with
} the above assumptions. 


\section{Matching}

The basic idea behind law application in SAOITHÍn is that the goal focus is matched against one or many laws. Those matches that succeed return a binding and a replacement template, and the user can then select which successful match to apply. The focus is replaced by the template, instantiated using the bindings. The matching we perform is basic structural matching of one test predicate against another treated as a pattern, with success returning a binding that matches pattern variables to test sub-predicates. We are not performing unification, either in isolation, or w.r.t. to some equational theory [BL98]. This is in contrast to Isabelle, for example, which uses higher-order unification [Pau88]. However, we do not simply match $F$ against all of $L$-if $L$ has certain forms, we can match against part of the law, offerring another part as a replacement:

$L$ is $P \equiv Q$ : If the law is an equivalence we look for matches against the LHS and RHS separately, offering the other side as a replacement.

$L$ is $A \Rightarrow C$ : Given an implication law, a partial match against either the antecedent or consequent is still useful. Using equational reasoning, a match against $A$ can offer $A \wedge C$ as a replacement, whilst a match against $C$ can be replaced by $C \vee A$.

So the upshot is that we can get a number of different types of matches against laws with a certain structure. We now proceed to discuss the process of matching.

\subsection{Basic Matching}

The first phase of matching is a basic structural comparison of a test predicate $Q$ (the goal focus) against a pattern predicate $P$ (part of a law), which either fails, or succeeds and returns an incomplete binding, mapping pattern meta-variables to corresponding fragments of the test predicate. A key feature to note is that pattern variables and meta-variables match anything of the appropriate class (expr/type/predicate), provided they are not "known". Remember, a name is "known" if it appears in any of the following theory tables: obs, typedef, exprdef or preddef. A known name can only match against either itself or its definition as found in those tables.

None of the above gives any clue as to why basic matching returns an incomplete binding. The reason for this lie in three areas: our use of explicit substitutions, the fact that ordering is not important in substitutions or quantifier lists, and our desire to support a lot of flexibility in matching quantifiers. The motivation for the latter stems from the fact that SAOITHín is designed to support foundational work in the UTP, and so we want to be able to derive very general laws. For example, we want to have general laws like:

$$
\left(\forall x, x_{1}, \ldots, x_{n} \bullet P\right) \equiv\left(\forall x_{1}, \ldots, x_{n} \bullet P\right), \quad x \notin P
$$

where we can somehow specify that $n \geq 0$ and avoid listing the $x_{i}$. Also, given the above law and the axiom

$$
\left(\exists x_{1}, \ldots, x_{n} \bullet P\right) \equiv \neg\left(\forall x_{1}, \ldots, x_{n} \bullet \neg P\right)
$$


we would like to be able to prove

$$
\left(\exists x, x_{1}, \ldots, x_{n} \bullet P\right) \equiv\left(\exists x_{1}, \ldots, x_{n} \bullet P\right), \quad x \notin P
$$

To this end, the syntax of quantifiers in the logic of SAOITHÍn is in fact more complex than suggested so far. Instead we view a quantifier as being followed by two comma-separated lists of variables, with a semicolon separating the two lists:

$$
\begin{array}{ll}
\forall x_{1}, \ldots, x_{m} ; x s_{1}, \ldots, x s_{n} \bullet P & m \text { s-qvars, } n \text { m-qvars } \\
\forall x_{1}, \ldots, x_{m} \bullet P & m \text { s-qvars, } 0 \text { m-qvars } \\
\forall ; x s_{1}, \ldots, x s_{n} \bullet P & 0 \text { s-qvars, } n \text { m-qvars }
\end{array}
$$

Either list may be empty. The first list is of so-called "single" quantifier variables (s-qvars), each of which corresponds to a conventional quantifier variable, and, when occurring in a pattern predicate, are required to match distinct s-qvars from the test predicate. The second list is of "multiple" quantifier variables ( $m$-qvars), which are intended to represent many quantifier variables. A m-qvar in a pattern can match any mixture of s-qvars and m-qvars, including none. Matching is now complicated by the fact that the ordering of s-qvars, m-qvars is irrelevant, so in principle we have to try every possible permutation. Basic matching will succeed for simple cases, basically those with only one pattern qvar, but otherwise will return a deferred match in the form of a test-qvars/pattern-qvars pair, in addition to any bindings from the quantifier bodies. The basic matching is then followed by two phases, one that tries to resolve the deferred matches, and then a step to check the side-conditions. Both of these phases need to know about the free variables of the goal.

\subsection{Free Variables}

Many laws have side-conditions attached, all of which are conditions regarding the free-variables. However, the presence of explicit meta-variables and substitution complicates the calculation of the free variables of a predicate. To see this, we first present some clauses of the definition of free variables of a predicate:

$$
\begin{aligned}
\mathrm{fv} e & =\text { all variables free in } e \\
\mathrm{fv}\left(P_{1} \wedge P_{2}\right) & =\left(\mathrm{fv} P_{1}\right) \cup\left(\mathrm{fv} P_{2}\right) \\
\mathrm{fv}(\forall \vec{v} \bullet e) & =(\mathrm{fv} e) \backslash \vec{v} \\
\mathrm{fv} M & =\mathrm{fv} . M \\
\mathrm{fv} P\left[e_{1}, \ldots, e_{n} / v_{1}, \ldots, v_{n}\right]= & (\mathrm{fv} P) \backslash\left\{v_{1}, \ldots, v_{n}\right\} \\
& \cup \bigcup\left\{i: 1 \ldots n \mid v_{i} \in \mathrm{fv} P \bullet \mathrm{fv} e_{i}\right\}
\end{aligned}
$$

We see that for meta-variables we need to return the fact that computation of their free variables needs to be held until they are instantiated at some later point in time. With substitution, we see that the resulting set is contingent upon the freeness or otherwise in $P$ of the substitution target variables $\left(v_{1}, \ldots, v_{n}\right)$. The upshot of all this is that we need to represent the free-variables of predicates using 
a free-variable set-expression, rather than just a simple variable enumeration. The required syntax of such expressions is as follows:

$$
\begin{aligned}
& s \in \operatorname{SetExpr}::=\left\{v_{1}, \ldots, v_{n}\right\} \quad \text { Enumeration, } n \geq 0 \\
& \text { f } \mathrm{f} . M \quad \text { Meta free-variables } \\
& s_{2} \backslash\left\{v_{1}, \ldots, v_{n}\right\} \text { Set Difference, } n \geq 0 \\
& \text { | } \bigcup\left(s_{1}, \ldots, s_{n}\right) \quad \text { Union, } n \geq 0 \\
& \text { | } m \Rightarrow s \quad \text { Conditional Set } \\
& m \in \text { Member }::=a \in s \quad \text { Element Membership } \\
& \text { | } \bigwedge\left(m_{1}, \ldots, m_{n}\right) \text { Conjunction, } n \geq 2
\end{aligned}
$$

Most are self explanatory, except conditional set membership $m \Rightarrow s$, which denotes set $s$ if $m$ is true, otherwise is empty.

A further complication arises with the user-defined language constructs. The free variables of these can only be established by expanding out their definitions. If we were simply to report "cannot tell" for the free variables of such constructs, then we would be unable to match any law involving a user-construct that had a side-condition, and would be forced to expand them out as an explicit proof step in any case. As one of the aims of UTP is to support laws at the language level, without always having to expand out the underlying predicate form, this is not a viable solution.

Accordingly, the algorithm for computing free variables expands language definitions on-the-fly, to get to a predicate form to establish its free-variables. This is why every language construct has to have defining laws with the name and form described in $\S 5$. This on-the-fly activity is invisible to the user, occurring behind the scenes. This explains why the language definition laws cannot be mutually recursive, otherwise this procedure could not be guaranteed to terminate.

\subsection{Match Completion}

Once a basic match is done, we want to try to complete it by using context information to figure out a suitable qvar matching. The context information we use includes the bindings already obtained, some which may pre-determine how qvars should match, as well as any information regarding the free variables of the focus, as well as side-conditions for both the goal and the relevant law. This process is quite complex and we omit any further details, apart form noting that in complicated cases it may fail to find a valid matching even if one exists. However there is a work-around by using other proof steps to re-organise quantifiers until matching does work. For a similar reason, the checking of $\alpha$-equivalence used to terminate a proof is also incomplete and may fail to detect such an equivalence - the same work-around can be used here as well.

Finally, we check that the law side-condition, if any, when translated in terms of the goal using the bindings, is actually a consequence of the goal sidecondition, if any: $s c_{\text {law }} \wedge s c_{\text {goal }} \equiv s c_{\text {goal }}$. This is achieved by code that automatically evaluates/simplifies a side-condition using free-variable set-expressions 


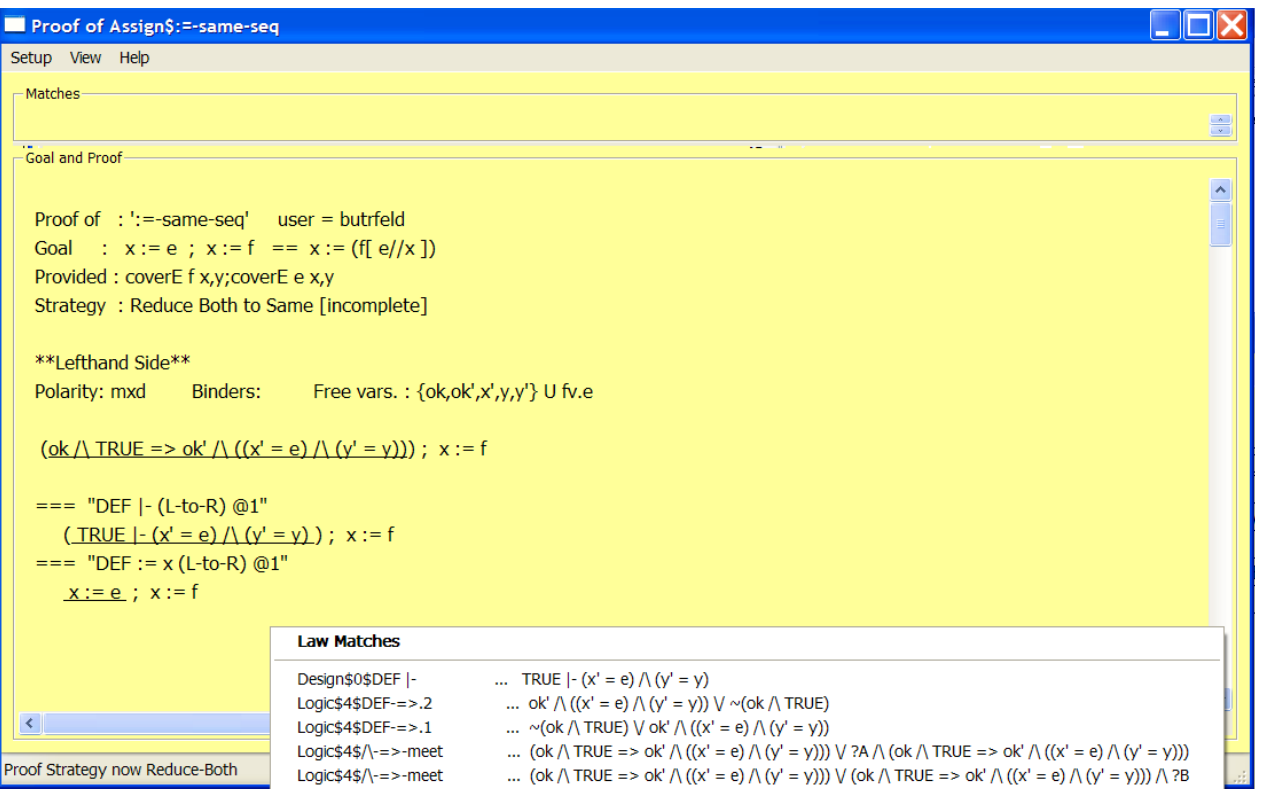

Fig. 1. Saоithín Proof Example

and then establishes that the above equivalence holds. This is facilitated by the existence of a normal-form for side-conditions.

\section{Usage}

Here we show a proof (that $x:=e ; x:=f=x:=f[e / x]$ ), using the reduce both sides strategy (Fig.1). A number of steps have already been performed, expanding the definitions of $:=$ and $\vdash$, with the steps shown in reverse order (first proof step last). The user has right-clicked, bringing up a possible list of replacements. Law matching can return a large number of successful matches, and these need to be presented to the user in a manner that makes it easy for them to select the law they wish to apply. There are basically two ways for a user to request law matching: one displays all the matches in a special window for the user to peruse, whilst the other displays up to 20 of them in a popup menu for immediate selection. In both cases we need some way to rank matches so the most useful appear first. In essence, we need some form of heuristics that can take a law match and compute a ranking number. There has been a limited amount of experimentation in this area, and it is a difficult area to get right. While at present the heuristics are hard-coded, we expect to improve matters to give the users more control in this area in future. The performance of the law matching is excellent at present, with a virtually instant return of results, while the GUI performance can be a little slow when inspecting large theory tables, so this is an obvious area for improvement. 
Earlier versions of SAOITHÍn have been tested on 3rd-year Computer Science students at TCD who have taken an elective module on Formal Methods, which presents the subject through the UTP framework. Initially they were shown the use of the prover using a logic module with initial laws drawn from the axiomatization of equational logic in [GS94], material that they studied in their first year. Then they were asked to prove conjectures arising from useful library theories, such as Sets or Lists. Most problems reported by the students had to do with installation or user-interface behaviour issues - most students found the proof aspects easy to apply once these initial hurdles were overcome, and feedback from them has been used to improve the tool. We have used the tool to produce a wide range of proofs of UTP relevance, of which the following is just a short representative list:

$$
\begin{array}{cc}
(\sigma \frown \tau)-\sigma=\tau & S_{1} \cap\left(S_{2} \cup S_{3}\right)=\left(S_{1} \cap S_{2}\right) \cup\left(S_{1} \cap S_{3}\right) \\
P \vdash Q \equiv P \vdash P \wedge Q \quad & x:=e ; y:=f \equiv y:=f[e / x] ; x:=e, \quad y \notin e \\
\mathbf{R 3}(\mathbf{R 3}(P)) \equiv \mathbf{R 3} & \mathbf{R 2} \circ \mathbf{R 2}=\mathbf{R 2}
\end{array}
$$

\section{Conclusions and Future Work}

We have given motivation for, and presented, SAorTHín, a theorem-proving assistant for UTP. The current state of the tool is still experimental, with considerable scope for enhancement and improvement, but is already a useful tool for experienced $^{6}$ developers of UTP foundations.

Apart from the obvious need for comprehensive library of useful theorems from general logic, arithmetic, set, list and map theory, there are still a few foundational issues that need to be resolved. The current limitation to fixed alphabets is too restrictive, but we have a plan, using special meta-variables known to the pattern matcher, to be able to describe generic laws that cross a number of theories: the gold standard here would be a definition of sequential composition

$$
P ; Q \hat{=} \exists O B S_{m} \bullet P\left[O B S_{m} / O B S^{\prime}\right] \wedge Q\left[O B S_{m} / O B S\right], \quad O B S_{m} \text { fresh }
$$

that works in any theory whose observations are homogenous (meta variables $O B S_{m}, O B S^{\prime}$ and $O B S$ would need special pattern matching and binding treatment). The treatment of undefinedness in a way that limits its impact on general proving is still under exploration, and we are exploring various ideas in this area [Art96]. Given the existing work on UTP and Circus using Proofpower-Z, we also hope to explore the ability to take the GUI front-end of SAOITHín, and couple it to ProofPower-Z as a backend, given some appropriate transformation of how proofs and theorems are presented.

\footnotetext{
${ }^{6}$ Not too trusting!
} 


\subsection{Acknowledgments \& Availability}

We would like to thank Colm Bhandal, Karen Forde, Simon Dardis, Jim Woodcock and the Formal Methods classes of 2009 and 2010 for their work on, and feedback about, SAOIthín. SAOITHÍn is written in Haskell [JHA $\left.{ }^{+} 99\right]$, managed using the distributed source-code manager Mercurial [O'S09], and is released open-source under GPL v2, at http://www. scss.tcd.ie/Andrew.Butterfield/Saoithin/. The version available at the time of writing is $0.86 \alpha 9$.

\section{References}

[Art96] R. D. Arthan. Undefinedness in Z: Issues for specification and proof. In William Farmer, Manfred Kerber, and Michael Kohlhase, editors, Proc. Mechanization of Partial Functions Workshop, affiliated to CADE-13, New Brunswick, pages 3-12, 1996.

[Asp00] David Aspinall. Proof general: A generic tool for proof development. In Susanne Graf and Michael I. Schwartzbach, editors, TACAS, volume 1785 of $L N C S$, pages 38-42. Springer, 2000.

[BC04] Yves Bertot and P. (Pierre) Castéran. Interactive theorem proving and program development: Coq'Art: the calculus of inductive constructions. Texts in theoretical computer science. Springer Verlag, 2004.

[BKT94] Yves Bertot, Gilles Kahn, and Laurent Théry. Proof by pointing. In Masami Hagiya and John C. Mitchell, editors, Theoretical Aspects of Computer Software, International Conference TACS '94, Sendai, Japan, April 19-22, 1994, Proceedings, volume 789 of LNCS, pages 141-160. Springer, 1994.

[BL98] F. Baader and M. Leucker. Comparison of two semantic approaches to unification. In Proceedings of the 12th International Workshop on Unification, number SI-98/8 in Research Report, Universita di Roma, La Sapienza, June 1998.

$\left[\mathrm{GHH}^{+} 95\right]$ C. George, A. E. Haxthausen, S. Hughes, R. Milne, S. Prehn, and J. S. Pedersen. The RAISE Development Method. The BCS Practitioners Series. Prentice Hall Int., 1995.

[GS94] David Gries and Fred B. Schneider. A Logical Approach to Discrete Math. Texts and Monographs in Computer Science. Springer-Verlag, New York, NY, 1994.

[HH98] C. A. R. Hoare and Jifeng He. Unifying Theories of Programming. Prentice-Hall, 1998.

[Hoa85] C. A. R. Hoare. Programs are predicates. In Proc. of a discussion meeting of the Royal Society of London on Mathematical logic and programming languages, pages 141-155, Upper Saddle River, NJ, USA, 1985. PrenticeHall, Inc.

$\left[\mathrm{JHA}^{+}\right.$99] S. Peyton Jones, J. Hughes, L. Augustsson, D. Barton, B. Boutel, W. Burton, J. Fasel, K. Hammond, R. Hinze, P. Hudak, T. Johnsson, M. Jones, J. Launchbury, E. Meijer, J. Peterson, A. Reid, C. Runciman, and P. Wadler. Report on the programming language haskell 98, February 1999. http://haskell.org/onlinereport/.

[MS89] Carrol Morgan and J. W. Sanders. Laws of the Logical Calculi. Oxford University Computing Laboratory, Programming Research Group, 1989. 
[MU05] Petra Malik and Mark Utting. CZT: A framework for Z tools. In Helen Treharne, Steve King, Martin C. Henson, and Steve A. Schneider, editors, $Z B$ 2005: Formal Specification and Development in $Z$ and B, volume 3455 of $L N C S$, pages 65-84. Springer, 2005.

[NPW02] Tobias Nipkow, Lawrence C. Paulson, and Markus Wenzel. Isabelle/HOL A Proof Assistant for Higher-Order Logic, volume 2283 of LNCS. Springer, 2002.

[NW06] Gift Nuka and Jim Woodcock. Mechanising a unifying theory. In Steve Dunne and Bill Stoddart, editors, Unifying Theories of Programming, First International Symposium, UTP 2006, volume 4010 of LNCS, pages 217235. Springer, 2006.

[OCW09] Marcel Oliveira, Ana Cavalcanti, and Jim Woodcock. A UTP semantics for circus. Formal Asp. Comput, 21(1-2):3-32, 2009.

[O'S09] Bryan O'Sullivan. Mercurial: The Definitive Guide. O'Reilly Media, June 2009.

[Pau88] Lawrence C Paulson. The foundation of a generic theorem prover. Technical Report TR-130, Computer Laboratory, University of Cambridge, March 1988.

[Sha96] Natarajan Shankar. PVS: Combining specification, proof checking, and model checking. In Mandayam K. Srivas and Albert John Camilleri, editors, Formal Methods in Computer-Aided Design, First International Conference, FMCAD '96, volume 1166 of $L N C S$, pages 257-264. Springer, 1996.

[Tou01] George Tourlakis. On the soundness and completeness of equational predicate logics. J. Log. Comput., 11(4):623-653, 2001.

[Wie06] Freek Wiedijk, editor. The Seventeen Provers of the World, Foreword by Dana S. Scott, volume 3600 of Lecture Notes in Computer Science. Springer, 2006.

[ZC09] Frank Zeyda and Ana Cavalcanti. Mechanical reasoning about families of UTP theories. Electr. Notes Theor. Comput. Sci, 240:239-257, 2009. 